Documento No. 2

\title{
EL FINANCIAMIENTO DEL GASTO PÚBLICO EN UNA ECONOMÍCA EN CRECIMIENTO: \\ EL CASO DE MÉXICO
}

\author{
Por \\ Alain Ize
}

Noviembre, 1978

Versión Preliminar. No citar en publicaciones sin permiso del autor. Este documento forma parte de un proyecto conjunto. El Colegio de México-Banco de México

$\underline{\text { Las ideas contenidas en el presente ensayo son responsabilidad exclusiva del autor y no reflejan la }}$ posición del Banco de México 


\title{
EL FINANCIAMIENTO DEL GASTO PÚBLICO EN UNA ECONOMÍA EN CRECIMIENTO: EL CASO DE MÉXICO
}

\author{
Por \\ A. Iza*
}

El gasto público puede ser financiado a través de cuatro canales principales. El más usual es a través de la imposición fiscal, el segundo corresponde al endeudamiento interno, el tercero al endeudamiento externo y el cuarto a la emisión primaria de circulante. Pero sólo los dos primeros están bajo el control directo del gobierno en un contexto de largo plazo (por lo menos teóricamente); el endeudamiento externo está restringido por la condición de equilibrio de la balanza de pagos y por el ritmo de crecimiento ${ }^{1}$, mientras que si se quiere evitar el financiamiento inflacionario, el aumento del circulante está también limitado por la tasa de crecimiento de la economía.

La composición y evolución de las fuentes de financiamiento del gasto público federal en México durante los cuatro últimos sexenios aparece en el Cuadro 1. Como se puede apreciar, el endeudamiento interno como proporción del ingreso nacional -realizado principalmente a través del encaje legal-, ha incrementado su importancia relativa. El mayor uso de este instrumento, a partir de la segunda mitad de los años cincuenta, ha sido considerado favorablemente debido a que vino a sustituir gradualmente a la expansión primaria como principal fuente de financiamiento del gasto público ${ }^{1}$. Sin embargo, a partir del último sexenio, algunas voces se elevaron en contra de una tasa de encaje legal que se estimaba había llegado a niveles demasiado elevados, restringiendo por lo tanto los fondos disponibles para la inversión privada y/o promoviendo la sustitución de fondos internos por fondos externos, acelerándose así el ritmo de crecimiento de la deuda privada externa. En el presente sexenio, y quizás como resultante de esta corriente de opiniones, se ha reducido en cierta medida la tasa de encaje legal promedio. Sin embargo, la polémica en torno al impacto del financiamiento por endeudamiento interno continúa, y se escuchan opiniones a veces radicalmente opuestas sobre la conveniencia de utilizarlo como fuente de financiamiento del gasto público.

* El autor agradece la valiosa asistencia de José Dávila y Angel Calderón, y la ayuda brindada por Guillermo Ortiz y Armando Baqueiro. Este trabajo se ha visto también beneficiado por diversos comentarios hechos en los seminarios internos tanto del Banco de México como del Colegio de México.

Aún suponiendo que no haya limitaciones en la cantidad de créditos externos que se puedan obtener, es necesario suponer en un contexto de largo plazo que las reservas en divisas deban crecer al mismo ritmo que la economía.

1 Cf. Brothers y Solís (1968). 


\section{Cuadro 1}

Fuente de Financiamiento del Gasto

Público Federal

(Como proporción del PIB)

\begin{tabular}{lrrrr}
\hline & $\begin{array}{c}\text { Impuestos a } \\
\text { Ingresos } \\
\text { Propios }\end{array}$ & $\begin{array}{c}\text { Financiamiento } \\
\text { por emisión } \\
\text { primaria } \\
\text { PIB }\end{array}$ & $\begin{array}{c}\text { Financiamiento } \\
\text { por Encaje } \\
\text { PIB }\end{array}$ & $\begin{array}{c}\text { Deuda } \\
\text { Externa }\end{array}$ \\
\hline $1953-1958$ & .088 & .00253 & .0000 & N. d. \\
$1959-1964$ & .075 & .00199 & .0049 & N. d. \\
$1965-1970$ & .092 & .00219 & .0144 & .0021 \\
$1971-1975$ & .113 & .00812 & .0248 & .0026 \\
\hline
\end{tabular}

FUENTE: Banco de México, S.A., Estadísticas NAFINASA y estimaciones del autor

El objetivo de este trabajo es de ubicar la discusión en torno al financiamiento del gasto público dentro del marco general de un modelo de crecimiento, comparando el impacto de un incremento o en la presión fiscal o en la tasa de encaje legal en tres dimensiones de la economía mexicana: el tamaño del sector público (ligado a consideraciones estructurales y de bienestar social), el crecimiento de la economía (o sea u ritmo de desarrollo en términos cuantitativos) y el tamaño de la deuda externa (determinantes esencial de la dependencia de la economía vis a vis del exterior). Dependiendo de la magnitud de algunos parámetros esenciales (como la propensión al consumo, la magnitud de la deuda externa y la productividad del gasto público) se identifican varias zonas con diferentes características e implicaciones en cuanto a políticas óptimas de financiamiento.

En una primera parte se discuten en forma general las características del sistema de encaje legal en México, y se analizan sus posibles repercusiones sobre la economía. El modelo teórico utilizado se desarrolla en la segunda parte, mientras que en la tercera se presentan algunas estimaciones de los parámetros en el caso de México, y se derivan algunas conclusiones tentativas de política económica.

II. El Encaje y el Papel del Sistema Financiero.

II.1 La Mecánica del Encaje Legal en México

El papel tradicional de la tasa de reserva bancaria como instrumento de política monetaria, es el de estabilización económica a corto plazo, a través de su influencia sobre el multiplicador 
bancario. Sin embargo, en el caso de México, debido a la escasa importancia de instrumentos de captación directa del ahorro interno, el encaje legal ha sido usado a la vez como mecanismo de captación de fondos para el sector público y como herramienta de control macroeconómico a corto plazo.

Un esquema aproximado de flujos de fondos en la economía mexicana sería entonces el que se muestra en la gráfica 1. El Banco Central crea dinero y lo transfiere al gobierno (flujo $\left.\dot{M}_{s}\right)$, al ser gastado ese dinero recae en poder del público quien lo deposita en parte en el sistema financiero. Si $K$ es la proporción de monedas y billetes a depósitos y $e$ la tasa de encaje legal, es fácil demostrar que al terminar el proceso de expansión crediticia, un flujo $(1-\mathrm{e})^{\dot{\mathrm{M}}_{\mathrm{s}}} / \mathrm{K}$ de nuevos créditos ha sido canalizado hacia el público mientras que el flujo de fondos captado a través del encaje ha sido de $e^{\dot{\mathrm{M}}_{\mathrm{s}} / \mathrm{K}}$. La cantidad adicional de monedas y billetes en poder del público es $\dot{M}_{s}$ mientras que el aumento de pasivos del Banco Central es $\dot{M}_{s} / K$. El financiamiento total obtenido por el gobierno, $\dot{M}_{s}(1+\mathrm{e} / \mathrm{K})$, corresponde al ahorro neto del público que ha sido conservado o en circulante o en depósitos y no prestado otra vez al mismo público:

$$
\dot{M}_{s}\left(1+\mathrm{e} / \mathrm{K}=\mathrm{M}_{\mathrm{s}}+\dot{\mathrm{M}}_{s} / \mathrm{K}-(1-\mathrm{e}) \dot{\mathrm{M}}_{\mathrm{s}} / \mathrm{K}\right)
$$


Gráfica 1

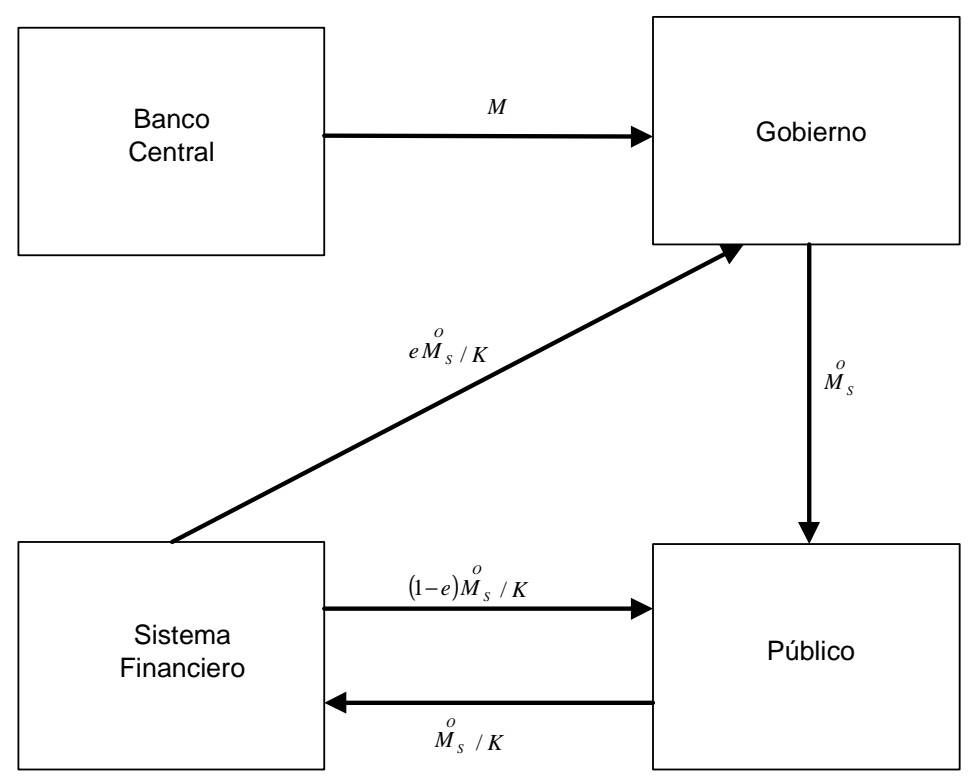

II. El Papel de la Intermediación Financiera.

Más allá de su función de facilitar los pagos y de mantener los saldos del público, el papel básico de cualquier sistema financiero es el de la canalización constante de recursos ociosos hacia las necesidades de inversión. Aún si se argumenta que no existe a menuda una clara distinción física entre ahorradores y desahorradores (ya que una buena parte de los ahorros los realizan las mismas empresas y empresarios), es menos objetable que deba en general existir una distinción temporal entre empresas que tienen en un momento dado excesos de fondos y empresas que al estar invirtiendo tienen necesidades netas de fondos. El sistema financiero puede hacer circular esos fondos de unas a otras.

Si $\quad V$ es la velocidad anual de rotación de los créditos al sector privado, el volumen de créditos rotatorios extendidos durante ese lapso de tiempo es $(1-\mathrm{e}) \mathrm{V} \mathrm{M}_{2} / \mathrm{K} \cdot$ Comparado al volumen de nuevos créditos, $(1-$ e $) \dot{\mathrm{M}}_{\mathrm{s}} / \mathrm{K}$, es fácil checar que la relación entre créditos rotatorios y nuevos 
créditos es de $V / g$, en donde $g$ es la tasa de aumento del circulante. Bajo cualquier hipótesis razonable de órdenes de magnitud sobre $V$ y $\quad g$ se llega entonces a la conclusión que la parte más importante del financiamiento al sector privado radica en los créditos rotatorios, o sea en la función de transmisión de fondos desde empresas con excedentes hasta empresas con déficits ${ }^{1}{ }^{1}$ más que en la función de canalización de recursos de ahorradores netos hacia desahorradores netos.

\section{II.3 Posibles impactos de la restricción crediticia.}

Una alza en la tasa de encaje restringe entonces el crédito al sector privado en dos maneras: a través de una reducción en el flujo: $(1-$ e $) \stackrel{\dot{M}_{s} / K}{\mathrm{~K}}$ y a través de una reducción en el monto total de créditos rotatorios extendidos durante el año (el efecto acervo: $(1-\mathrm{e}) \dot{\mathrm{M}}_{s} \mathrm{~V} / \mathrm{K}$ ). De modo que no sólo ocurre un proceso de desviación del ahorro, sino que también se da un fenómeno de desintermediación financiera.

El impacto probable sobre la inversión privada es difícil de estimar a priori ya que está estrechamente relacionado con varios aspectos estructurales. En primer lugar, es posible pueda sustituir el financiamiento bancario por mayores recursos internos, pero esto implica que se deban posponer inversiones hasta que se hayan almacenado los fondos requeridos para realizarlas ${ }^{2 /}$ Esta mayor lentitud en el proceso de inversión significa necesariamente una baja en el ritmo de inversión a nivel macroeconómico.

En segundo lugar, es posible que la empresa tenga acceso a mercados financieros extranjeros y pueda por lo tanto financiarse externamente. El aumento correspondiente de la deuda externa puede sin embargo ser indeseable al aumentar la dependencia del país con respecto del exterior. La substitución de fondos internos por fondos externos puede además favorecer indebidamente a las empresas multinacionales quienes tienen un acceso más directo a los mercados exteriores.

\footnotetext{
1/ O también de consumidores que están ahorrando para la compra de bienes duraderos hacia consumidores que están justamente realizando esa compra.

2/ En un universo perfectamente previsible y determinista, las empresas podían planear sus inversiones e ir acumulando los fondos necesarios con el adelanto suficiente; la falta de financiamiento externo es mucho más crítica en un contexto estocástico.
} 
En tercer lugar, la restricción crediticia puede favorecer fuertemente un proceso de concentración industrial dado que las empresas grandes se ven necesariamente favorecidas para conseguir los pocos créditos existentes, y dado que pueden repartir internamente en forma más eficiente sus recursos disponibles.

Finalmente, es difícil predecir cómo se distribuye el impacto de una restricción crediticia sobre el consumo, la inversión productiva y la inversión improductiva. Es posible que la propensión al consumo se reduzca en tiempos de escasez de fondos; primero porque los empresarios sustituyen la falta de préstamos por un mayor esfuerzo de financiamiento interno; segundo, porque los créditos que están destinados a financiar la adquisición de bienes durables se ven fuertemente frenados.

Es claro entonces que la escasez de fondos puede tener un impacto o bien sobre la inversión privada productiva, o bien sobre el consumo privado, o bien sobre el endeudamiento externo. El impacto global sobre la economía de un aumento en la tasa de encaje debería entonces depender de las elasticidades de estas funciones. Esto es lo que vamos ahora a analizar en más detalle.

\section{Un Modelo de Financiamiento a Largo Plazo.}

\section{III.1 Hipótesis Generales del Modelo.}

Se considera una economía en crecimiento uniforme dentro de un contexto de largo plazo. Se puede suponer que existe un constante pleno empleo de la capacidad instalada, más no de la mano de obra, de manera que la tasa de crecimiento está condicionada por el ritmo de inversión. Se supone que el nivel de precios es fijo, haciéndose así abstracción de las posibilidades de financiamiento inflacionario del gasto público. La tasa de cambio es un parámetro exógeno manejable por el gobierno pero se considera constante una vez que se ha fijado. El déficit en cuenta corriente está, por su parte, definido como un residuo que permite ajustar la demanda y la oferta de bienes internos. Un exceso de demanda interna se traduce por lo tanto en un incremento de las importaciones y una reducción de las exportaciones ${ }^{\underline{1}}$

1/ G. Ortiz (1977) desarrolló un modelo de crecimiento con características similares, aunque su enfoque es distinto. 
El gasto público tiene dos componentes: uno directamente productivo que permite incrementar el acervo total de capital, y otro que se refleja en un aumento de la productividad de la inversión, tanto privada como pública, a través de su impacto sobre la educación, salud, infraestructura, etc.

Por último, se considera que todo el endeudamiento interno se efectúa a través del encaje $\mathrm{e}^{\underline{2}}$ y que se pagan intereses $r_{p}$ sobre esa deuda. El método general que se seguirá consistirá en comparar trayectorias de crecimiento obtenidas a partir de diferentes tasas de encaje y de imposición fiscal. No se analizarán las posibles trayectorias de ajuste de una trayectoria de largo plazo a otra ${ }^{3 /}$

\section{III.2 Condiciones de Equilibrio.}

El sector real, la condición de equilibrio entre oferta y demanda totales de bienes es:

$$
Y+\mathrm{Z}=\mathrm{C}+\mathrm{I}+\mathrm{G}
$$

es donde $Y$ representa la oferta interna de bienes y servicios, $Z$ corresponde al déficit en cuenta corriente (importaciones menos exportaciones), $C$ es el consumo privado, $I$ la inversión privada y $G$ el gasto público.

El sector monetario el equilibrio entre oferta y demanda de dinero se puede escribir en forma Fisheriana:

$$
Y=\mathrm{M}_{\mathrm{s}} \mathrm{V} .
$$

En el sector externo, el equilibrio en balanza de pagos se expresa como:

$$
\stackrel{\circ}{D}_{P}+\dot{\mathrm{D}}_{\mathrm{G}}=\mathrm{M}_{\mathrm{R}}+\mathrm{Z}+\mathrm{r}_{\mathrm{E}}\left(\mathrm{D}_{\mathrm{p}}+\mathrm{D}_{\mathrm{G}}\right)
$$

\footnotetext{
2/ La captación directa de ahorros internos tendría prácticamente el mismo impacto macroeconómico que el sistema de encaje. Se puede por lo tanto considerar un solo canal de financiamiento.

3/ O sea las trayectorias de "traverse" según la terminología de Kicks. (1960).
} 
en donde $D_{p}$ y $\quad D_{G}$ corresponden respectivamente a los acervos de deudas externas contraidas por los sectores privados y públicos, $r_{E}$ es la tasa de interés externa y $M_{F}$ es la reserva en divisas del Banco Central.

Finalmente en el sector gobierno, el equilibrio entre ingresos y egresos es:

$$
G+\mathrm{r}_{\mathrm{p}} \mathrm{e}^{\mathrm{M}_{\mathrm{s}} / \mathrm{K}}+\mathrm{r}_{\mathrm{E}} \mathrm{D}_{\mathrm{G}}+\stackrel{\circ}{\mathrm{M}}_{\mathrm{R}}=\mathrm{T}+\dot{\circ}_{\mathrm{s}}(1+\mathrm{e} / \mathrm{K})+\stackrel{\circ}{\mathrm{D}}_{\mathrm{G}}
$$

de manera que el gasto público más el pago de intereses sobre la deuda interna y externa más el incremento en reservas de divisas, pueden ser financiados a través de impuestos $(T)$, o a través de creación primaria de circulante $\left(\stackrel{\circ}{M}_{s}\right)$, o mediante el flujo de encaje $\left(\stackrel{\circ}{M}_{s}\right.$ e $\left./ \mathrm{K}\right)$, o del incremento de la deuda externa $\stackrel{\circ}{D}_{G}$.

A las anteriores condiciones de equilibrio puede añadirse una ecuación de crecimiento a través de lo cual el incremento en el ingreso es función de la inversión privada y de la parte productiva del gasto público:

$$
\stackrel{\circ}{Y}=\alpha(\mathrm{I}+\mu \mathrm{G})
$$

En donde $\mu$ es un coeficiente que permite medir la productividad del gasto público en comparación con la productividad de la inversión privada.

III.3 Ecuaciones Definicionales y de Comportamiento.

Se definen las siguientes variables:

$$
t=\mathrm{T} / \mathrm{Y}
$$




$$
\begin{aligned}
& 1=\mathrm{D} / \mathrm{Y}, \mathrm{D}=\mathrm{D}_{\mathrm{p}}+\mathrm{D}_{\mathrm{G}} \\
& \lambda=\mathrm{G} / \mathrm{Y} \\
& g=\stackrel{\mathrm{Y}}{\mathrm{Y}} \\
& \gamma=\mathrm{M}_{\mathrm{R}} / \mathrm{Y} ; \\
& \gamma \text { es tomado como un parámetro dado. }
\end{aligned}
$$

Se hacen además las hipótesis de comportamiento siguientes:

Se supone que el consumo privado es función del ingreso disponible y que la propensión al consumo se ve afectada por la escasez de fondos, medida ésta por una variable $r$ que correspondería a la tasa de interés prevaleciente si el mercado de fondos prestables estuviera siempre en equilibrio. Si, como en el caso de México, este mercado no está siempre en equilibrio, $r$ es un índice que refleja al mismo tiempo el costo de los fondos y su escasez relativa en términos de excesos de demanda o de oferta. Para simplificar, no consideraremos inicialmente los efectos que tengan sobre el ingreso disponible los ingresos y egresos que corresponden al pago de intereses sobre deudas. En ese caso, la función consumo se escribe simplemente:

$$
C=\mathrm{c}(\mathrm{r}) \mathrm{Y}(1-\mathrm{t}), \mathrm{c}^{\prime} \leq 0
$$

En la misma forma la inversión privada es función del ingreso y de la tasa de interés. Se supone por ahora que no se ve afectada por la presión fiscal, para más adelante examinar el caso en que sí lo es.

$$
I=\mathrm{i}(\mathrm{r}) \mathrm{Y}, \quad \mathrm{i}^{\prime} \leq 0 .
$$

Dado que el flujo de fondos externos privados, debería también depender de la escasez interna de fondos: 


$$
\stackrel{\circ}{D}_{\mathrm{p}}=\mathrm{d}(\mathrm{r}) \mathrm{Y}, \quad \mathrm{d}^{\prime} \leq 0 \text {. }
$$

Tanto la velocidad de circulación, $V$, como el multiplicador bancario, $K$, se consideran constantes $^{3 /}$

Para terminar de describir el modelo sólo se necesitan añadir las condiciones de crecimiento uniforme:

$$
\mathrm{g}=\dot{\mathrm{Y}} / \mathrm{Y}=\stackrel{\circ}{\mathrm{D}_{\mathrm{p}} / \mathrm{D}_{\mathrm{p}}}=\stackrel{\circ}{\mathrm{M}_{\mathrm{s}}} / \mathrm{M}_{\mathrm{s}}
$$

III.4 El sistema reducido.

Dividiendo entre $Y$ las ecuaciones (1) a $\left(5\right.$, y eliminando las variables $z / y \quad$ y $\quad D_{\mathrm{G}} / \mathrm{Y}$, se obtiene el siguiente sistema reducido:

$$
\begin{aligned}
& (1-\mathrm{t})(1-\mathrm{c})+\mathrm{d}\left(1-\mathrm{r}_{\mathrm{e}} / \mathrm{g}\right)=\mathrm{i}+(1+\mathrm{e} / \mathrm{k}) \mathrm{g} / \mathrm{V}-\mathrm{r}_{\mathrm{p}} \mathrm{e} / \mathrm{KV} \\
& 1\left(\mathrm{~g}-\mathrm{r}_{\mathrm{e}}\right)=\gamma+\mathrm{c}(1-\mathrm{t})+\mathrm{i}+\lambda-1 \\
& g=\alpha(\mathrm{i}+\mu \lambda)
\end{aligned}
$$

Este sistema consta de tres ecuaciones y de cuatro variables endógenas, $g, \lambda, 1 \mathrm{y} \mathrm{r}$, de modo que sobra un grado de libertad. Es claro que ese grado de libertad proviene de la ecuación de equilibrio en el mercado de vienes (ecuación (1)) y se debe a que el déficit en cuenta corriente, $Z$, fue definido como un residuo. No se puede entonces saber a priori, por ejemplo, sin un incremento de $G$ repercutirá en el corto plazo esencialmente en una baja de la demanda privada interna $(C+\mathrm{I})$ o en una alza del déficit en cuenta corriente $(Z)$. En vez de fijar arbitrariamente la forma en que $Z$ varía,

3/ Esta hipótesis, dentro de un contexto de largo plazo, parece razonable para el caso de México. 
preferimos dejar un grado de libertad en el modelo, dejando así cierto margen exógeno en las políticas compensatorias para alterar el déficit en cuenta corriente (a través de medidas fiscales, de variaciones en la tasa de cambio o de reformas estructurales). El impacto de las variaciones en $e$ o en $t$ podrá repartirse entonces entre la deuda externa, el crecimiento y la participación del sector público, en una forma hasta cierto punto exógena que dependerá de las medidas tomadas para contrarrestar el déficit en cuenta corriente. La dirección y las características generales de dicho impacto podrán, sin embargo, ser definidas de manera específica.

\section{III.5 El Sistema Diferenciado.}

Diferenciado el sistema (15) al (17), eliminando los términos en $d r$ y reagrupando el resto, se obtiene un sistema de la forma:

$$
\left[\begin{array}{cccc}
g-\mathrm{r}_{\mathrm{E}} & 1-1_{\lambda} & 0 \\
\mathrm{~g}-\mathrm{r}_{\mathrm{E}} & 0 & \frac{\Omega}{\nabla} & \left(1-1^{\prime \prime}{ }_{\lambda}\right) \\
0 & - & 0 & \Omega
\end{array}\right]\left[\begin{array}{l}
d_{1} \\
d_{g} \\
d_{\lambda}
\end{array}\right]\left[\begin{array}{ll}
\mathrm{A}_{\mathrm{t}}^{\circ}\left(\mathrm{c}-\mathrm{c}_{\mathrm{t}}\right) & \mathrm{A}_{\mathrm{e}}^{\circ}\left(\mu-\mu_{\mathrm{e}}\right) \\
\mathrm{A}_{\mathrm{t}}^{1}\left(\mathrm{c}-\mathrm{c}_{\mathrm{t}}\right) & \mathrm{A}_{\mathrm{e}}^{1}\left(1_{\lambda}-1\right) \\
-A_{t}^{2} & -\mathrm{A}_{\mathrm{e}}^{2}
\end{array}\right]\left[\begin{array}{l}
d_{t} \\
d_{e}
\end{array}\right]
$$

Los coeficientes $1_{\lambda}, 1_{\lambda}^{\prime}, 1_{\lambda}^{\prime \prime}, \mathrm{c}_{\mathrm{t}}, \mathrm{c}_{\mathrm{t}}^{\prime} \mathrm{y} \mu_{\mathrm{e}}$ son valores críticos de las variables 1 , с y $\mu$; los efectos de cambios en la presión fiscal o el encaje legal ( $t$ y e) sobre las variables que caracterizan el estado de crecimiento uniforme de la economía $(g, \lambda, 1)$, dependen en cuanto a sus signos, de la magnitud de estas variables $(1, \mathrm{c}, \mu)$ con respecto a sus valores críticos $\left(1_{\lambda}, 1_{\lambda}^{\prime} 1_{\lambda}^{\prime \prime} \mathrm{c}_{\mathrm{t}}, \mathrm{c}_{\mathrm{t}}^{\prime} \mathrm{y} \mu_{\mathrm{e}}\right)$. Estos coeficientes son:

$$
\begin{aligned}
& 1_{\lambda}=\frac{1}{\alpha \mu}-\frac{\Psi\left(\mu-\mu_{\mathrm{e}}\right)}{\mu\left(1+\mu_{\mathrm{e}} \Phi\right)} \\
& 1_{\lambda}^{\prime}=\frac{1}{\alpha \mu_{\mathrm{E}}} \\
& 1_{\lambda}^{\prime \prime}=1_{\lambda}^{\prime}-\frac{\nabla}{\Omega}\left(\mu-\mu_{\mathrm{E}}\right)
\end{aligned}
$$




$$
\begin{aligned}
& \mu_{E}=\frac{\mathrm{i}^{\prime}}{\mathrm{i}^{\prime}+\mathrm{c}^{\prime}(1-\mathrm{t})} \\
& c_{t}=\frac{\mu_{\mathrm{e}}-\mu}{\mu_{\mathrm{e}}-(1+\mu \phi)} \\
& c_{t}^{\prime}=\frac{1-1_{\lambda}^{\prime}}{1+\mathrm{W}+\mathrm{Z} / \alpha}
\end{aligned}
$$

donde:

$$
\begin{aligned}
& \psi=(1+\mathrm{e} / \mathrm{K}) 1 / \mathrm{V}-\mathrm{r}_{\mathrm{e}} \mathrm{d} / \mathrm{g}^{2} \\
& \phi=\frac{\mathrm{d}^{\prime}\left(1-\mathrm{r}_{\mathrm{E}} / \mathrm{g}\right)}{\mathrm{i}^{\prime}}
\end{aligned}
$$

El resto de los coeficientes de la ecuación (18) que determinan la magnitud de los cambios ejercidos en $g, \lambda$ y 1 por cambios en $t$ y e son:

$$
\begin{aligned}
& \nabla=\Psi+\phi / \alpha+1 / \alpha \mu_{\mathrm{e}} \\
& \Omega=\mu\left(1+\mu_{\mathrm{e}} \phi\right) / \mu_{\mathrm{e}} \\
& A_{t}^{\circ}=\frac{\mu_{e}(1+\mu \phi)}{\mu\left(1+\mu_{\mathrm{e}} \phi\right)} \\
& A_{e}^{\circ}=\frac{\mathrm{g}-\mathrm{r}_{\mathrm{p}}}{\mathrm{KV} \mathrm{V}_{\mu}\left(1+\mu_{\mathrm{e}} \phi\right)} \\
& A_{1 t}=\frac{1+\Psi+\phi / \alpha}{\nabla}
\end{aligned}
$$




$$
\begin{aligned}
& A_{e}=\frac{\mathrm{g}-\mathrm{r}_{\mathrm{p}}}{\mathrm{KV}\left(\Psi+\phi / \alpha+1 / \alpha \mu_{\mathrm{e}}\right)} \\
& A_{t}^{2}=1-\mathrm{c} \\
& A_{e}^{2}=\left(\mathrm{g}-\mathrm{r}_{\mathrm{p}}\right) / \mathrm{KV}
\end{aligned}
$$

Dados los signos de $c^{\prime}$, i' y d' es fácil cerciorarse de que si $\Psi$ es positivo y $g$ es mayor a $r_{p}$ y a $\mathrm{r}_{\mathrm{E}}$, los coeficientes (27) a (34) son positivos; en el apéndice se demuestra asimismo que aún si $\Psi$ es negativo, los coeficientes serían positivos.

\section{III.6 Condiciones de Vialidad.}

A partir del sistema de ecuaciones de la sección anterior, (y para valores de $t$ y e dados), puede demostrarse que cuando:

$$
\begin{gathered}
g \rightarrow \mathrm{r}_{\mathrm{E}} \quad, \quad 1 \rightarrow \circ \circ \\
\mathrm{y} \text { si } 1 \rightarrow 1_{\lambda} \quad, \quad \mathrm{g} \rightarrow \circ \circ \quad \mathrm{y} \quad \lambda \rightarrow \circ \circ .
\end{gathered}
$$

El límite $r_{E}$ indica que para poder sostener la deuda externa, es necesario que

$$
g>\mathrm{r}_{\mathrm{E}}
$$

esto es, que el ritmo de crecimiento de la economía sea mayor que la tasa de interés pagada sobre la deuda externa.

La segunda condición, 
expresa que el país no puede endeudarse más allá de un cierto límite. Al acercarse a ese límite, el ritmo de crecimiento y la participación del sector público en la economía crecen indefinidamente, debido a que el flujo creciente de deuda pública externa permite incrementar sin límites el gasto y la inversión pública. Es claro que esta es una situación que difícilmente se podría alcanzar, dadas las condiciones existentes sobre la capacidad de endeudamiento ${ }^{4 /}$ que delimita una zona en la cual la economía debe permanecer.

Nótese también que si $g<\mathrm{r}_{\mathrm{p}}$, los coeficientes $A_{e}^{\circ} \mathrm{A}_{\mathrm{e}}^{1}$ de la ecuación (18) se vuelven negativos. $\mathrm{Si} A_{e}^{\circ}$ es negativo, un incremento en el encaje $(d e>0)$ para un nivel dado de endeudamiento y de deuda externa $(d t=\mathrm{dl}=0)$, implicaría una reducción en el ritmo de crecimiento de la economía $\left(d_{g}<0\right)$ a menos que el gasto público sea muy improductivo $\left(\mu<\mu_{\mathrm{e}}\right)$. La razón de este resultado es clara: si la tasa de crecimiento es menor a la tasa de interés interna, un incremento en el encaje implica una erogación del gobierno mayor que el ingreso percibido por concepto del aumento en el propio encaje, de manera que el gasto público necesariamente disminuye y, por consiguiente, el flujo de inversión privada puede incrementarse y con ella la tasa de crecimiento. Por ello, para que el encaje sea utilizable como fuente de financiamiento del gasto público, es necesario que se cumpla la siguiente condición:

$$
g>\mathrm{r}_{\mathrm{p}}
$$

\section{III.7 Regiones.}

El impacto sobre $1, \lambda$ y g de una variación en el encaje o en la imposición fiscal dependerá entonces, como se puede observar en el sistema de ecuaciones (18), del valor de los

4/ Por otra parte, es probable que la productividad marginal del gasto público tendería rápidamente hacia cero al aumentar demasiado rápidamente su monto. Se puede entonces apreciar que si $\mu \mu$ tendiera hacia cero, $1_{\lambda}$ (ecuación (19)) tendería hacia el infinito y no habría entonces límite de endeudamiento. 
parámetros, $c, \mu$ y 1 con respecto a los valores límites $c_{t}, \mu_{\mathrm{e}}, \mathrm{c}_{\mathrm{t}}^{\prime} \mathrm{y} 1_{\lambda}^{\prime}$. Se pueden así identificar distintas zonas en espacio $(c, \mu, 1)$ en las cuales la reacción de la economía a variaciones en los instrumentos de política mencionados tendrá características propias; este mapa aparece en la gráfica 2. Se aprecia que existen 6 regiones posibles correspondiendo a diferentes valores de $c, \mu$ y 1 .

Analizaremos primero como se comporta la economía en cada una de estas regiones, para después examinar cómo varía la posición y el tamaño de esas regiones conforme cambian los parámetros del modelo.

a) Región I (OEFGDA): $0 \leq \mu \leq \mu_{\mathrm{E}} \leq 1$

$$
\begin{aligned}
& 0 \leq 1 \leq 1_{\lambda} \leq 1_{\lambda}^{\prime} \\
& c_{\mathrm{t}}^{\prime} \leq 0 \leq \mathrm{c} \leq \mathrm{c}_{\mathrm{t}} \leq 1
\end{aligned}
$$

Esta región corresponde a valores bajos tanto de la propensión al consumo como de la productividad del gasto público y de la deuda externa.

A partir de la primera ecuación en el sistema (18) se pude deducir que tanto un aumento en los impuestos como en el encaje legal tienden a reducir el ritmo de crecimiento y/o aumentar la deuda externa. No se puede entonces conservar el mismo ritmo de crecimiento sin aumentar la deuda externa o conservar el mismo nivel de deuda sin reducir el ritmo de crecimiento. A partir de la segunda ecuación del mismo sistema se puede, sin embargo, deducir que tanto un incremento en $e$ como en $t$ permiten elevar $\lambda$. El problema en esta región es entonces que se puede elevar la participación del sector público en la economía, pero es a costa de un menor crecimiento o de un mayor endeudamiento externo.

No es difícil ver cuales son las razones económicas de este dilema: el sector público siendo improductivo y la propensión al consumo siendo baja, restar fondos al sector privado implica una baja sustancial en el ritmo de inversión productiva y por lo tanto de la tasa de crecimiento. Esta baja se puede compensar mediante un mayor endeudamiento externo que permita proporcionar fondos al sector privado y acelerar la inversión estatal.

b) Región II (ABCDEF): $\quad 0 \leq \mu \leq \mu_{\mathrm{E}} \leq 1$ 


$$
\begin{aligned}
& 0 \leq 1 \leq 1_{\lambda} \leq 1_{\lambda}^{\prime} \\
& c_{\mathrm{t}}^{\prime} \leq 0 \leq \mathrm{c}_{\mathrm{t}} \leq \mathrm{c} \leq 1
\end{aligned}
$$

\section{Gráfica 2}

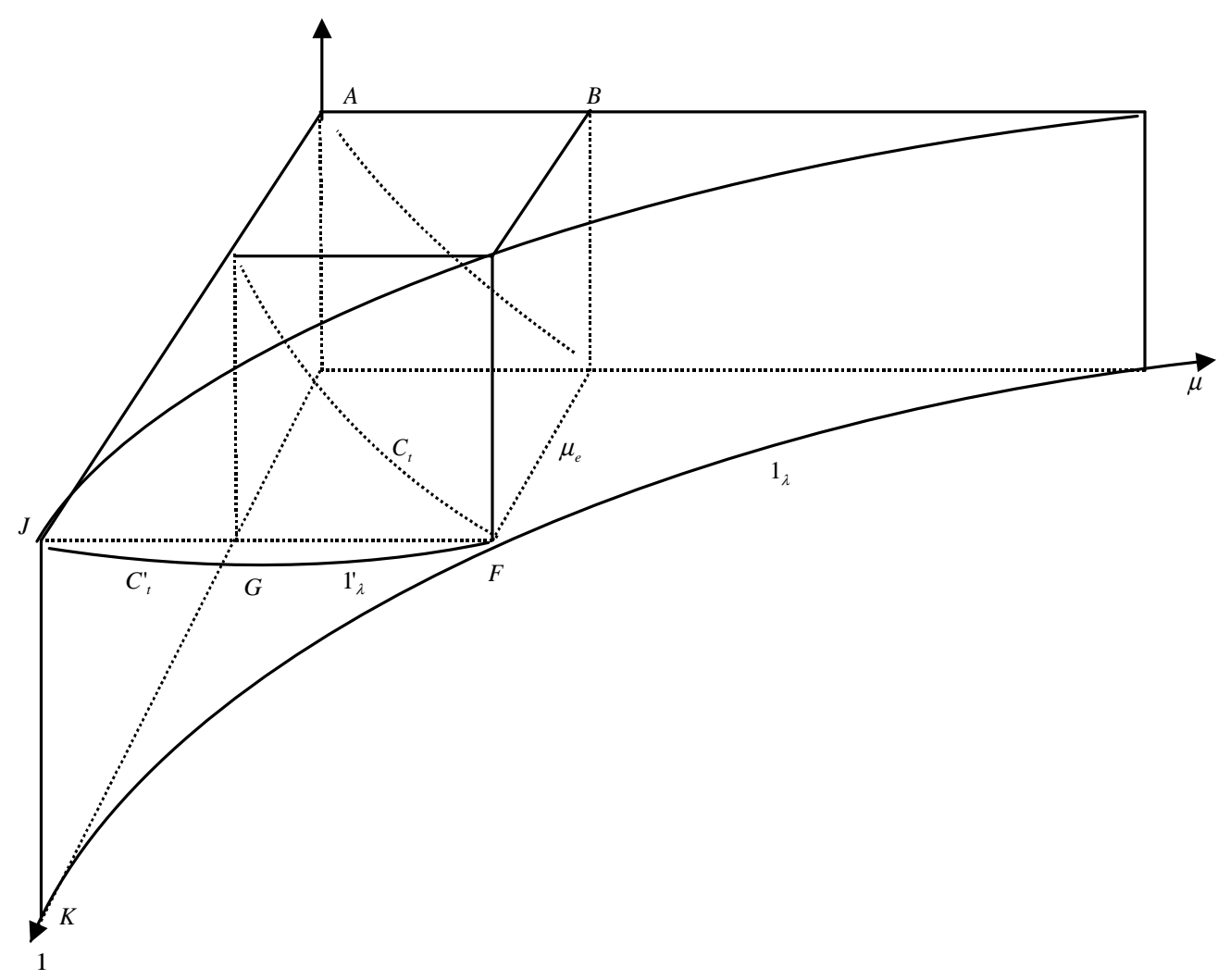

La única diferencia con respecto a la región anterior es que la propensión al consumo es mayor. Por lo tanto, como se puede apreciar en la misma ecuación del sistema diferenciado, una alza en los impuestos al frenar sustancialmente el consumo y reorientar los fondos correspondientes hacia la inversión estatal (aún poco productiva), permite elevar el ritmo de crecimiento y/o bajar el endeudamiento externo.

La posible baja en el endeudamiento externo se puede explicar fácilmente; regresando a la ecuación (3) y dividiéndola entre $Y$, se obtiene la siguiente ecuación: 


$$
1=(\mathrm{z}+\gamma) /\left(\mathrm{g}-\mathrm{r}_{\mathrm{E}}\right)
$$

en donde $z=\mathrm{Z} / \mathrm{Y}$.

Examinando esta ecuación, puede observarse que cualquier incremento en el ritmo de crecimiento que no se acompañe de una alza en el déficit en cuanta corriente permite reducir la deuda externa en relación con el producto interno, disminuyendo así el peso de la deuda. Inversamente, un menor crecimiento implica una carga mayor de la deuda externa.

Las implicaciones en cuanto a política económica son claras en esta región: es deseable reducir lo más posible el encaje y aumentar al máximo la presión fiscal; se alcanzará así una mayor participación del estado en la economía, una mayor tasa de crecimiento y una menor deuda externa.

c) Región III (FCBEHI): $\quad 0 \leq \mu_{e} \leq \mu \leq 1$

$$
\begin{aligned}
& 0 \leq 1 \leq 1_{\lambda} \leq 1_{\lambda}^{\prime} \\
& c_{\mathrm{t}}^{\prime} \leq 0 \leq \mathrm{c}_{\mathrm{t}} \leq \mathrm{c} \leq 1
\end{aligned}
$$

La diferencia con respecto a la región anterior, es ahora que la productividad del gasto público es mayor. La primera ecuación es (18) permite entonces comprobar que tanto un aumento en el encaje como en la presión fiscal, tendrían un impacto positivo sobre el crecimiento y frenarían la deuda externa. Es claro que en este caso, el gasto público siendo muy productivo, conviene incrementar la participación del sector público en la economía a través de aumentos en el encaje o en los impuestos.

d) Región IV (GFDJ): $0 \leq \mu \leq \mu_{e} \leq 1$

$$
\begin{aligned}
& 0 \leq 1_{\lambda}^{\prime} \leq 1 \leq 1_{\lambda} \\
& 0_{\mathrm{t}}^{\prime} \leq \mathrm{c}_{\mathrm{t}}^{\prime} \leq \mathrm{c} \leq \mathrm{c}_{\mathrm{t}}
\end{aligned}
$$

Esta región tiene las mismas características que la región I salvo que 1 es ahora mayor que el valor crítico $1_{\lambda}^{\prime}$. A partir de la segunda ecuación en (18) se puede entonces deducir que 
mientras un incremento en $t$ con 1 constante permite todavía incrementar la participación del sector público en la economía $(\lambda)$, un aumento en $e$ tiene un efecto opuesto. O sea que en esta región un incremento en el encaje que no aumente la deuda externa, no sólo reduce el crecimiento sino que también reduce la participación del sector público. Las conclusiones de política son entonces claras: si se quiere realmente aumentar $\lambda$ (aunque sea en detrimento del crecimiento), esto se puede realizar a base de un incremento en la presión fiscal pero no subiendo el encaje legal; de manera que es necesario sustituir $e$ por $t$ tanto como se pueda.

La razón de este resultado se aprecia al notar que el flujo de capitales externos es proporcional a la tasa de crecimiento y al nivel de deuda externa:

$$
\stackrel{\circ}{\mathrm{D}}=\mathrm{g} \frac{\mathrm{D}}{\mathrm{Y}}=\mathrm{g} 1 \mathrm{Y}
$$

Si 1 es alto ( y fijo ), una reducción en $g$ causada por una alza en el encaje, implica una fuerte disminución en el flujo de endeudamiento público externo y, por lo tanto, una reducción en $G / Y$.

e) Región V (JLCE):

$$
\begin{aligned}
& 0 \leq \mu \leq \mu_{e} \leq 1 \\
& 0 \leq 1_{\lambda}^{\prime} \leq 1 \leq 1_{\lambda} \\
& 0 \leq \mathrm{c} \leq \mathrm{c}_{\mathrm{t}}^{\prime} \leq \mathrm{c}_{\mathrm{t}}
\end{aligned}
$$

Esta región se diferencia de la anterior por el hecho que $c$ es ahora menor que $c_{t}$. Utilizando las dos primeras ecuaciones del sistema diferenciado se puede mostrar que un incremento tanto de $e$ como de $t$ (que deja 1 constante), implica una reducción en el tamaño del sector público junto con una reducción en la tasa de crecimiento. Lo que ocurre es que debido a la menor propensión al consumo, a la baja productividad del gasto público y al elevado nivel de la deuda, cualquier desviación de fondos del sector privado influye negativamente sobre la tasa de crecimiento y también sobre la participación del sector público, ya que un menor crecimiento implica una menor capacidad de endeudamiento interno y externo. Resulta obvio que las implicaciones de política económica son: reducir tanto el encaje como la presión fiscal, para que se pueda financiar el gasto público a través del efecto multiplicador de un mayor crecimiento. 
f) Región V (JDCF): $\quad \begin{array}{ll}0 \leq \mu \leq \mu_{e} \leq 1 \\ 0 \leq 1_{\lambda}^{\prime} \leq 1 \leq 1_{\lambda} \\ 0 \leq \mathrm{c}_{\mathrm{t}}^{\prime} \leq \mathrm{c}_{\mathrm{t}} \leq \mathrm{c}\end{array}$

Esta última región está caracterizada por una alta propensión al consumo. A partir del sistema (189 se deduce que un aumento en $t$ conduciría a un incremento en $g$ y $/ \mathrm{o}$ una reducción en 1 así como un incremento en $\lambda$. Dado el impacto negativo del encaje (similar al de las dos regiones anteriores) es claro que hay que sustituirlo por impuestos. La recomendación aquí es en la misma dirección, pero de manera aún más clara que en el caso de la región II.

\section{III.8 Variaciones en los parámetros.}

Los valores límites más importantes en este modelo corresponden a los coeficientes $\mu_{e} \mathrm{y} \mathrm{c}_{\mathrm{t}}$; el valor de $1_{\lambda}^{\prime}$ se deriva a su vez inmediatamente del valor de $\mu_{e}$. Este último depende fundamentalmente de la relación de las derivadas $c^{\prime} / i^{\prime}$. Si esta relación es alta (o sea que el consumo se contrae más que la inversión en tiempos de escasez de fondos), $\mu_{e}$ será bajo y al menos que el gasto público sea muy poco productivo la economía se ubicara en la región III en la cual conviene incrementar la participación del estado o bien a través de impuestos o bien a través del encaje. En cambio si $c^{\prime} / i^{\prime}$ es bajo, (la inversión decrece más que el consumo cuando hay escasez de fondos), $\mu_{e}$ será alto, y lo más probable es que $\mu$ sea menor que $\mu_{e}$, siendo entonces de todas formas poco recomendable el uso del encaje.

En esta última situación, el hecho que se pueda o no elevar la presión fiscal depende entonces esencialmente del valor de $c$ comparado al de $c_{t}$. Puede observarse en la gráfica 3 (y en las ecuaciones (23) y (26)), que $c_{t}$ se reduce conforme aumentan la productividad del gasto público y la elasticidad con respecto a la tasa de interés del flujo de endeudamiento privado externo; una alza en $d^{\prime}$ aumenta la curvatura de $c_{t}$ y reduce la región I en beneficio de la región II. La razón es 
clara: un incremento en el flujo de deuda externa privada al compensar el efecto negativo ejercido por la captación gubernamental de fondos privados, permite mantener una alta tasa de inversión privada.

\section{Gráfica 3}

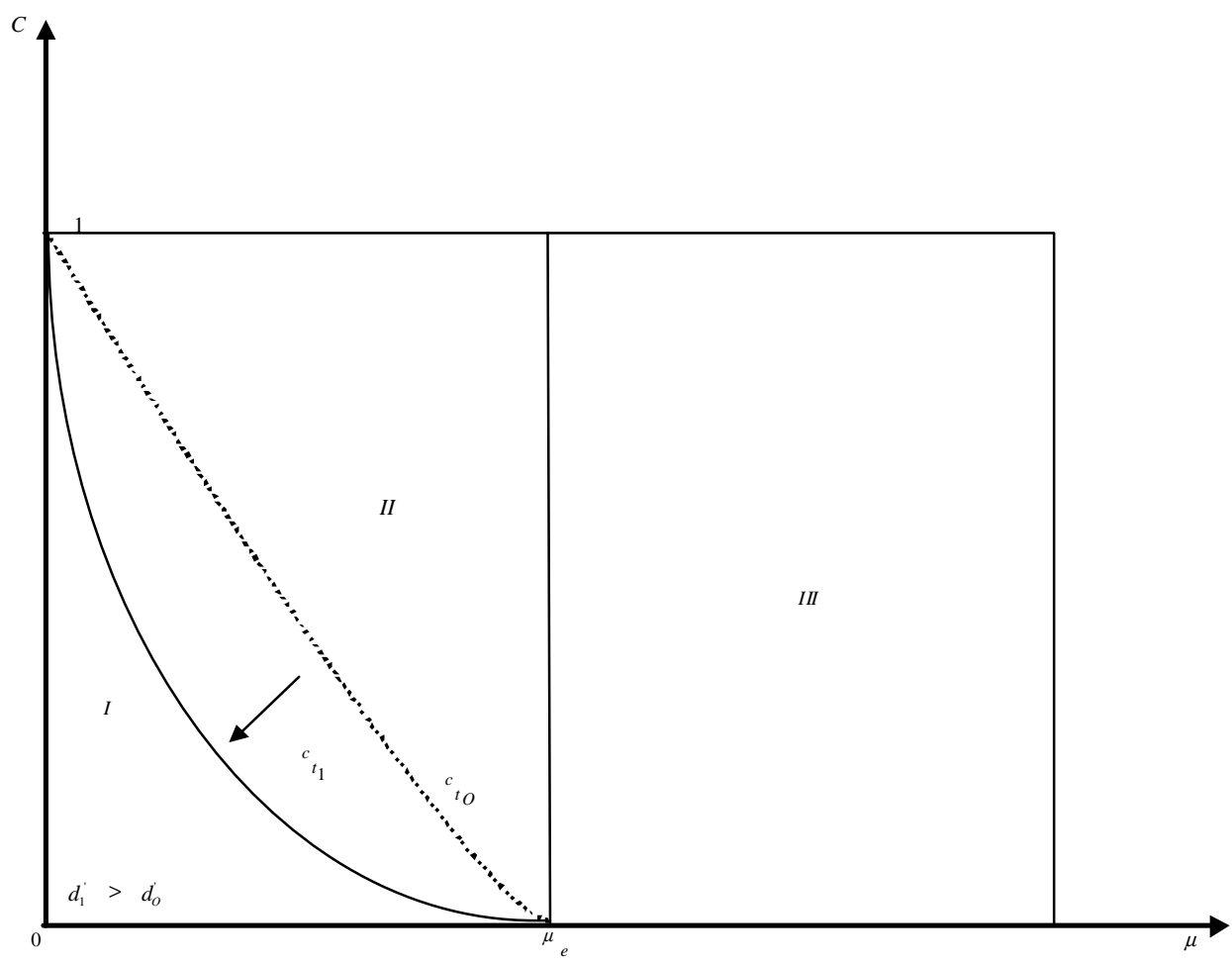

La magnitud de los efectos ejercidos por $e$ y $\mathrm{t}$ sobre $g, 1$ y $\lambda$ dependen, por otra parte, de los valores de los coeficientes $A^{\circ}$ de la ecuación (18). Se puede notar en particular que cuando $d^{\prime}$ tiene hacia el infinito, $\phi$ también tiende hacia el infinito y $A_{e}^{\circ} \mathrm{y} \mathrm{A}_{\mathrm{e}}^{1}$ tienden hacia cero. En el caso de perfecta movilidad de capitales, un cambio en el encaje no tiene ningún efecto directo sobre los objetivos $g, 1$ y $\lambda$, debido a que el sector privado puede sustituir íntegramente los fondos internos que se sustraen por fondos externos ${ }^{1 /}$

\section{III.9 Tres extensiones.}

Terminamos esta parte de desarrollo teórico del modelo con tres extensiones. La primera toma en cuenta el hecho que la inversión estatal no sólo incrementa el acervo total productivo sino que

\footnotetext{
1/ Sin embargo, una alza en el encaje sí permite aumentar $\lambda$; el sistema diferenciado (18) indica que este aumento
} provoca una alza en 1 y en $g$. 
también probablemente incrementa la productividad de la inversión no sólo pública sino también privada, a través de una mayor educación, salud, infraestructura, etc... La función de producción debería entonces ser de la forma:

$$
Y=\alpha\left(\frac{\mathrm{G}}{\mathrm{Y}}\right)(\mathrm{I}+\mu \mathrm{G}), \alpha^{\prime} \geq 0
$$

Al llevar a cabo esta modificación, y realizar los cálculos correspondientes, se encuentra que las diferencias esenciales con respecto al caso anterior son que, por una parte, el nuevo valor crítico $\mu_{e}$ es ahora menor:

$$
\mu=\mu_{\mathrm{E}}-\frac{\alpha}{\alpha^{2}} \mathrm{~g}
$$

y que, por la otra, el valor de $c_{t}$ para $\mu=0$ es ahora menor que uno. Como se puede entonces apreciar en la gráfica 4, el efecto de esta modificación es esencialmente de reducir el tamaño de las áreas I y II y ampliar el del área III; este resultado es lógico.

La segunda extensión consiste en suponer que un incremento en la presión fiscal no solamente reduzca el consumo a través de una reducción en el ingreso disponible, sino que también disminuya la inversión privada. Idealmente, se podrían diseñar impuestos que recaigan únicamente sobre el consumo y no sobre la inversión, pero esto puede ser difícil de realizar en la práctica. Examinaremos entonces el caso límite de un impuesto que afecta por igual el consumo y la inversión, de modo que la función de inversión es ahora:

$$
I=\mathrm{i}(\mathrm{r})(1-\mathrm{t}) \mathrm{Y}
$$

Se encuentra entonces que el nuevo valor crítico de $\mu_{e}$ es:

$$
\mu_{e}=\frac{\mathrm{i}^{\prime}}{c^{\prime}+\mathrm{i}^{\prime}}<\mu_{\mathrm{e}}
$$

y que $c_{t}$ es positivo para $\mu=\mu_{\mathrm{e}}$. El mapa en el espacio $(\mu, \mathrm{c})$ se transforma entonces en la forma indicada en la gráfica 5. Se observa claramente que los resultados más importantes son una 
reducción de la zona II, y la creación de una nueva zona (VII) en donde $\mu$ es mayor que $\mu_{e}$ y $c$ menor que $c_{t}$. O sea que se reduce el área en la cual es preferible financiar el gasto a través de los impuestos en vez del encaje y aparece una nueva área, para valores bajos de la propensión al consumo y valores no demasiado bajos de $\mu$, en la cual es mejor financiar el gasto a través del encaje que de los impuestos.

La última extensión consiste en incluir los pagos de intereses sobre las deudas internas y externas dentro de la definición del ingreso disponible. Dado que el público percibe ingresos sobre la deuda interna y tiene que pagar intereses sobre la deuda externa (privada), la nueva definición de ingreso disponible es:

$$
Y_{d}=\mathrm{Y}(1-\mathrm{t})+\mathrm{r}_{\mathrm{p}} \mathrm{e}^{\mathrm{M}_{\mathrm{s}} / \mathrm{K}}-\mathrm{r}_{\mathrm{e}} \dot{\mathrm{D}}_{\mathrm{p}}
$$

No detallaremos aquí los cálculos que permiten llegar a las nuevas expresiones de las constantes y de los valores críticos en el sistema (18). Sin embargo, el cambio más importante ocurre en los términos en de. Esté término en la primera ecuación del sistema (18) es ahora: 
Gráfica 4

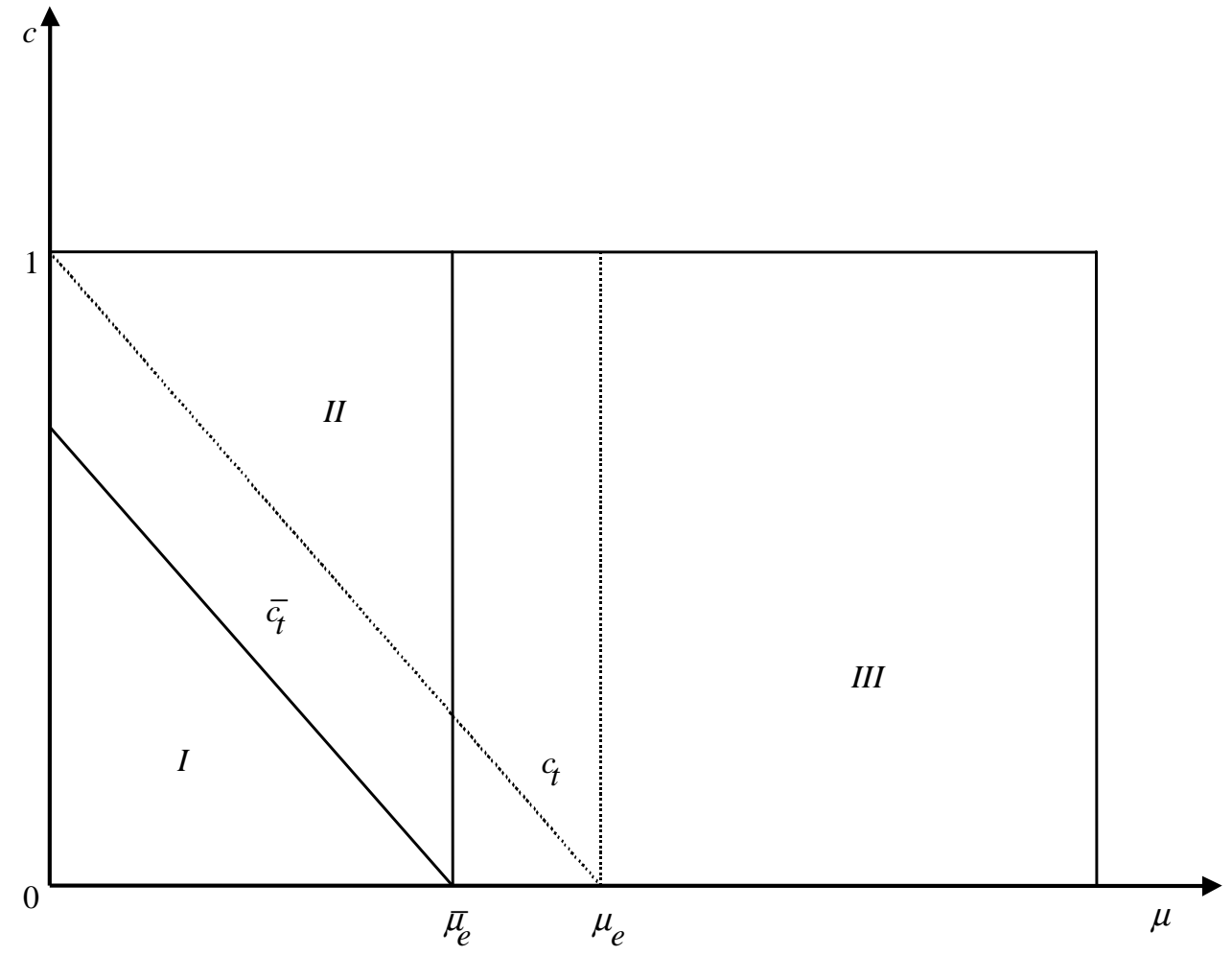

Gráfica 5

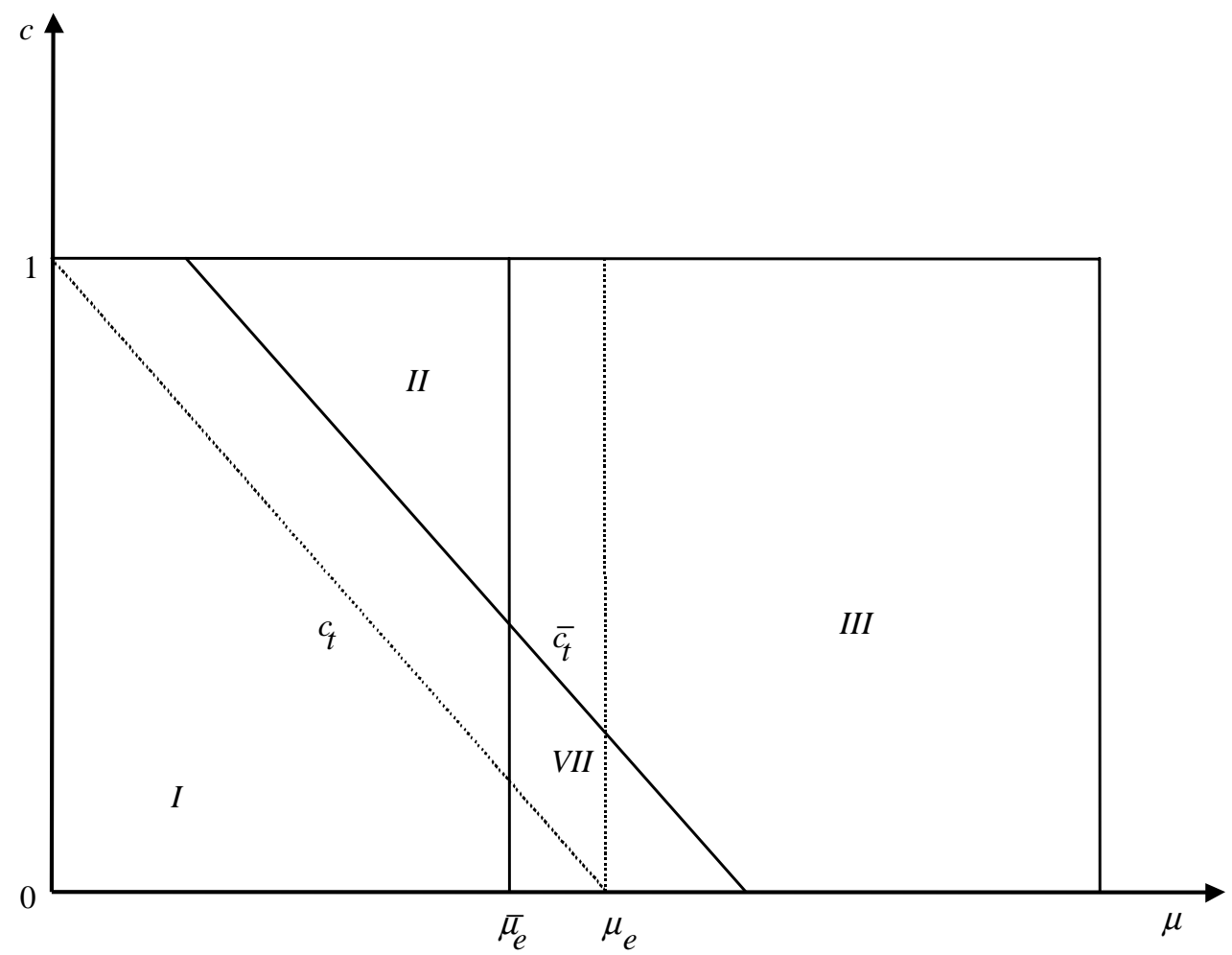




$$
\left[-A_{e}^{\circ}\left(\mu-\mu_{\mathrm{e}}\right)+\mathrm{r}_{\mathrm{p}} \mathrm{cA}_{\mathrm{t}}^{\circ}\right] \mathrm{de}
$$

en donde $\mu_{e}$ se redefine como:

$$
\mu_{e}=\frac{\mathrm{i}^{\prime}}{i^{\prime}+\mathrm{c}^{\prime}\left(1-\mathrm{t}+\mathrm{r}_{\mathrm{p}} \mathrm{e} / \mathrm{KV}-\mathrm{r}_{\mathrm{e}} \mathrm{cd}^{\prime} / g\right)}
$$

Como se puede apreciar en la ecuación (40), el efecto del pago de intereses sobre $r_{p}$ es esencialmente de reducir el margen dentro del cual un aumento en el encaje tiene un efecto positivo sobre la tasa de crecimiento; aun cuando $\mu$ es mayor a $\mu_{e}$, si $\mathrm{r}_{\mathrm{p}} \mathrm{cA}_{\mathrm{t}}^{\circ}>\mathrm{A}_{\mathrm{e}}^{\circ}\left(\mu-\mu_{e}\right)$, un incremento en el encaje tendrá un impacto negativo sobre la tasa de crecimiento. Esto se debe principalmente a que un alza en el encaje eventualmente eleva el consumo, debido a que ésta conlleva un incremento en el ingreso disponible del público.

El efecto del pago de intereses sobre la deuda externa depende, como se puede notar con la ecuación (41), de las elasticidades relativas del consumo y del flujo de capitales a un cambio en la tasa efectiva de interés. Si la elasticidad del flujo de capitales es mayor a la del consumo, el efecto de una alza en $r_{e}$ es de ampliar la zona en la cual un aumento en el encaje favorece el crecimiento del producto. Esto se explica porque al subir el encaje, el público sustituye fondos internos por fondos externos. Pero al hacerlo, tienen que reducir su consumo, dado que su ingreso disponible disminuye. Cuando mayor sea $r_{e}$, más grande es la baja en el consumo y también, por lo tanto, mayor el efecto positivo sobre el crecimiento ${ }^{\underline{1}}$.

Finalmente es interesante analizar cuál es el impacto del pago de intereses cuando hay perfecta sustitución de capitales internos por capitales externos. tomando el límite en (40) cuando $d$ ' tiende hacia el infinito, se encuentra el siguiente término:

$$
\left(-r_{e}-r_{p}\right) \frac{c}{K V} d e
$$

1/ Es claro, sin embargo, que una mayor tasa de interés externa implica un más alto nivel de deuda externa. 
Es fácil entonces deducir que para que el encaje sea utilizable, es necesario que la siguiente condición se cumpla:

$$
r_{p}<r_{\mathrm{e}}
$$

Este resultado es particularmente interesante debido a que contradice la condición generalmente requerida en los países en desarrollo, para que sea posible atraer capitales externos de que la tasa interna sea mayor a la tasa externa.

\section{Estimaciones.}

\section{IV.1 Metodología.}

Las magnitudes de las variables $t, \alpha, \mathrm{e} / \mathrm{K}, \mathrm{V}, \mathrm{r}_{\mathrm{p}}, \mathrm{r}_{\mathrm{e}}, \mathrm{c}, \mathrm{g}, 1 \mathrm{y} \mathrm{d}$ pueden ser estimadas sin mucha dificultad. Sin embargo, las derivadas $c^{\prime}, \mathrm{i}^{\prime}, \mathrm{y} \mathrm{d} \mathrm{d}^{\prime}$ y el coeficiente $\mu$, como se verá a continuación, presentan mayores problemas para su estimación, para lo que será necesario realizar un análisis de sensibilidad en torno a los parámetros $c^{\prime} / i^{\prime}, \mathrm{d} / i^{\prime}$ y $\mu$.

Después de calcular los diferentes valores críticos para poder ubicar así a la economía en la región que le corresponda, se estima el impacto que tendría el reemplazo total del endeudamiento interno por una mayor imposición fiscal. Se calcula la variación obtenida en cada una de las tres variables $1, \mathrm{~g}$ у $\lambda$, al dejar las otras dos constantes. Asimismo, se hacen estimaciones para el caso en el que los impuestos no tienen influencia sobre la inversión, y se repiten para el caso en el que sí la tienen. Debido a que las tasas promedio de interés real, tanto internas como externas son aproximadamente cero, no se tomaron en cuenta los efectos de pagos de intereses sobre el ingreso disponible. 
IV. 2 Fuentes estadísticas.

Los parámetros del modelo fueron estimadas como valores promedio de los años 70-75. La Tabla II indica sus magnitudes y la forma en que se calcularon ${ }^{1}$.

IV.3 Estimaciones de elasticidades y de la productividad de la inversión pública federal.

a) Liquidez y consumo.

Varios economistas han señalado que, en general, no existe equilibrio en el mercado de fondos prestables en México, sino que parece predominar una situación de racionamiento decrédito ${ }^{1}$. Esta situación de desequilibrio impide utilizar las tasas de interés sobre préstamos como indicadores de escasez de liquidez. En la medida en que exista racionamiento de crédito, es posible reemplazar la tasa de interés por la cantidad de créditos otorgados, representando esta última una restricción activa. Sin embargo, un serio problema que se presenta con estas estimaciones, es que la colinealidad entre las variables usadas como regresores puede ser muy importante.

Cuadro 2

\begin{tabular}{ccl}
\hline Variable & Valor & \multicolumn{1}{c}{ Descripción } \\
\hline$\alpha$ & .3 & Crecimiento del ingreso dividido entre inversión total \\
$V$ & 19.4 & Ingreso nominal dividido entre circulante \\
$K$ & .10 & Circulante dividido entre pasivos del sistema financiero \\
$e$ & .40 & Reservas divididas entre pasivos del sistema financiero \\
$c$ & .8 & Propensión al consumo \\
$i$ & .12 & Inversión privada dividida entre ingreso \\
$g$ & .123 & Tasa de crecimiento del ingreso (.063) más tasa promedio de \\
& .055 & Tasación mundial (.06) * rendimiento de los bonos en los Estados Unidos \\
$r_{e}$ & .06 & Tasa de rendimiento de los valores gubernamentales \\
$r_{p}$ & .37 & Deuda privada externa entre ingreso nominal (Deuda estimada en \\
$d$ & .75 & $\begin{array}{l}\text { Deuda externa total entre ingreso nominal (Deuda estimada en } 40 \\
\text { mil millones de dólares en 75) }\end{array}$ \\
1 & .11 & Recaudación fiscal entre ingreso nominal \\
$t$. & &
\end{tabular}

1/ Los datos provienen de NAFINSA: Statistics of the Mexican Economy, 1977, y de IMF: International Financial Statistics.

1/ Situaciones inversas de desequilibrio por exceso de oferta.

* En un contexto de largo plazo se puede suponer que la tasa de inflación interna es igual a la externa y que el tipo de cambio es fijo. Se obtendrían las mismas cifras con una inflación interna superior a la externa y un tipo de cambio deslizante que cubra el diferencial de tasas de inflación. 
Para el consumo, se estima, entre los años 50 - 76, la siguiente ecuación ${ }^{2 /}$

$$
c_{t}=.63 \mathrm{Yd}_{\mathrm{t}}+.46 \mathrm{~L}_{\mathrm{t}}+18760 \quad \mathrm{R}^{2}=.998
$$

Los valores entre paréntesis son desviaciones estándar, $Y d$ es el ingreso disponible y $L$ es el crédito total otorgado por el sistema financiero. Como se puede apreciar, en esta ecuación el crédito tiene un importante efecto positivo sobre el consumo. Este efecto puede, sin embargo, ser una simple resultante de la colinealidad existente entre las variables $\quad Y d$ y $L$ dado que el coeficiente de correlación entre estas dos variables es de .97. Con objeto de estimar mejor la importancia del efecto de colinealidad, se estimó la misma ecuación en primeras diferencias, encontrándose para esta regresión el resultado siguiente:

$$
\Delta \mathrm{c}_{\mathrm{t}}=.76 \Delta \mathrm{Yd}_{\mathrm{t}}-.04 \Delta \mathrm{L}_{\mathrm{t}}-138 \quad \mathrm{R}^{2}=.41
$$

En esta última ecuación, el término de crédito ya no es significativo y tiene además el signo opuesto del que previamente tenía, lo que sugiere que el valor de .46 encontrado en la ecuación anterior es, en todo caso, un límite superior del efecto real del crédito sobre el consumo ${ }^{\underline{1}}$

a) Inversión y liquidez.

La estimación de una función de inversión presenta problemas particularmente difíciles. El objetivo que aquí se persigue no es el de estimar la función de inversión más "correcta”, sino sólo el obtener algunos órdenes de magnitud sobre la importancia que pueda tener el crédito sobre la inversión privada. La función estimada fue del tipo usual:

2l Se estimó también una versión logarítmica de esta ecuación, pero no se obtuvieron mejores resultado que en la ecuación lineal.

11 Para tratar de evaluar mejor el efecto de colinealidad entre $Y d$ y $\mathrm{L}$, se estimaron también funciones del tipo $c_{t}-\alpha \mathrm{Yd}_{\mathrm{t}}=\beta \mathrm{L}_{\mathrm{t}}+\gamma$, haciendo variar $\alpha$ desde .6 hasta .8. Se encontró que el coeficiente $\beta$ bajó cuando $\alpha$ se elevó, y que pierde significancia cuando $\alpha$ es mayor a .72. Dado que la proposición al consumo en México parece ser mayor a .72; este resultado arroja también serias dudas sobre la validez de la ecuación en términos absolutos estimada anteriormente. 


$$
I_{t}=v\left(\mathrm{~L}_{\mathrm{t}}\right)\left[\widetilde{\mathrm{K}}_{\mathrm{t}+1}-(1-\delta)\right] \mathrm{K}_{\mathrm{t}}
$$

En donde $v$ es un coeficiente de ajuste que se hizo depender de la cantidad de créditos disponibles, $L_{t} ; \widetilde{\mathrm{K}}_{\mathrm{t}+1}$ es el acervo de capital deseado en el período $t+1$, fijado en base a ciertas expectativas de ingreso para ese período $\delta$ es un coeficiente de depreciación. El ingreso esperado para el período $t+1$ se estimó en la forma siguiente ${ }^{1 /}$

$$
\frac{\tilde{Y}_{t+1}-\tilde{Y}_{t}}{\tilde{Y}_{t}}=\frac{\tilde{Y}_{t}-Y_{t-1}}{Y_{t-1}}=\frac{Y_{t-1}-Y_{t-2}}{Y_{t-2}}
$$

La forma reducida de estas ecuaciones es: $\tilde{Y}_{\mathrm{t}+1}=\mathrm{Y}_{\mathrm{t}-1}^{3} / \mathrm{Y}_{\mathrm{t}-2}^{2}$. El acervo de capital fue estimado en base a la fórmula usual de inventarios perpetuos.

$$
K_{t}=\mathrm{I}_{\mathrm{t}}+(1-\delta) \mathrm{K}_{\mathrm{t}-1}
$$

Para formar un acervo de capital que refleje con mayor precisión el potencial productivo de la economía, se tomó como dato de inversión la inversión total en capital fijo, restándole la correspondiente a construcciones. Se escogió un coeficiente de depreciación de .1.

En vez de estimar de manera explícita una función de producción para relacionar el capital deseado al ingreso esperado en el período $t+1$, se realizó un simple análisis de tendencia de la relación capital / producto y se supuso que el capital deseado estaba sobre esa línea de tendencia $\underline{2 l}$

Con un coeficiente de depreciación de .1 la ecuación fue la siguiente:

$$
\log \mathrm{I}_{\mathrm{t}}=1.06+.62 \log _{\mathrm{t}}+.101 \log _{\mathrm{t}} \text {. }
$$

\footnotetext{
1/ Esta formulación refleja la hipótesis siguiente: las empresas invierten este año para satisfacer la demanda estimada el año próximo, a partir de las expectativas que se han formado en base al nivel de actividad económica alcanzado el año anterior.

2` Una descripción más completa del método usado y de los resultados se podrá encontrar en un trabajo del autor sobre la
} inflación en México. A. Ize (1978) 


$$
R^{2}=.97
$$

en donde $\quad W_{t}=\widetilde{\mathrm{K}}_{\mathrm{t}+1}-(1-\delta) \mathrm{K}_{\mathrm{t}}$.

A partir de esta ecuación, se podría concluir que el crédito tiene un importante efecto positivo sobre la inversión. Sin embargo, un examen más detallado pone en duda esta conclusión ya que la variable $W_{t}$, que refleja un efecto de aceleración, tiene a fluctuar mucho. A raíz de estas fluctuaciones, la variable $L_{t}$, que es más estable, tiende a captar la parte esencial de la tendencia normal de crecimiento de la inversión por lo que resulta muy significativa. El crédito sirve aquí como una variable que permite estabilizar el comportamiento de la inversión, frenándola en tiempos de auge económico $\left(W_{t}\right.$ alto $)$ y estimulándola en tiempos de depresión $\left(W_{t}\right.$ bajo $)$. El problema con el cual nos enfrentamos es el de definir una combinación "correcta" entre las variables de aceleración y de crédito. Si aumentamos el coeficiente de depreciación, la variable $W_{t}$ se vuelve más estable y como lo demuestran los resultados siguientes, el crédito pierde mucho de su importancia; con un coeficiente de depreciación de .8 se obtuvo la siguiente ecuación:

$$
\begin{aligned}
& \log \mathrm{I}_{\mathrm{t}}=.034+\underset{(.09)}{.24} \log \mathrm{L}_{\mathrm{t}}+.62 \log _{\mathrm{t}} . \\
& R^{2}=.98
\end{aligned}
$$

El crédito sigue teniendo un efecto positivo sobre la inversión pero su elasticidad es ahora solamente de .24 comparada con la cifra de .62 obtenida en el caso de $\delta=.1$. Se podría concluir tentativamente que el valor más apropiado de esta elasticidad probablemente se encuentra entre estos dos valores extremos $\underline{1 /}$

1/ Estos resultados son consistentes con las estimaciones de R. Montemayor (1974), quien usando la función de inversión del modelo Wharton, encuentra una elasticidad de la inversión con respecto al crédito de .50 . 
c) Liquidez y movimientos internacionales de capitales.

Con objeto de examinar el efecto del crédito interno sobre los movimientos de capitales, se estimó la siguiente regresión:

$$
\begin{aligned}
\dot{\mathrm{D}}_{\mathrm{t}} & =-1782-.019 \mathrm{~L}_{\mathrm{t}}+.01 \mathrm{Y}_{\mathrm{t}}+153\left(\mathrm{r}_{\mathrm{p}_{\mathrm{t}}}-\mathrm{r}_{\mathrm{e}_{\mathrm{t}}}\right)-34 \dot{\mathrm{p}} / \mathrm{p}_{\mathrm{t}} \\
(.007)(.002) & (164)
\end{aligned}
$$

$\dot{D}_{t}$ corresponde a los préstamos e inversiones extranjeras directas (excluyendo el crédito al gobierno), $Y_{t}$ es el ingreso nominal, $r_{p} \mathrm{r}_{\mathrm{e}}$ es el diferencial de tasas de interés $\mathrm{y} \quad \dot{p} / p$ es una variable que intenta captar las expectativas de devaluación en base al diferencial de índices de precios entre Estados Unidos y México. Se puede concluir que le crédito interno tiene un impacto negativo sobre los movimientos de capital que es significativo. Para tratar de disminuir el efecto de colinealidad entre $L$ y Y se estimó también esta ecuación de primeras diferencias, obteniéndose el siguiente resultado:

$$
\begin{aligned}
& A \dot{\mathrm{D}}_{\mathrm{t}}=414-.013 \Delta \mathrm{L}_{\mathrm{t}}+.00 \Delta \mathrm{Y}_{\mathrm{t}}-104 \Delta\left(\mathrm{r}_{\mathrm{p}_{\mathrm{t}}}-\mathrm{r}_{\mathrm{e}_{\mathrm{t}}}\right)-38 \Delta \dot{\mathrm{p}} / \mathrm{p}_{\mathrm{t}} \\
& \left.R^{2}=.029\right) \quad(.004)
\end{aligned}
$$

La variable de crédito sigue teniendo un signo negativo y un valor aproximadamente igual al obtenido en la ecuación anterior, pero resulta no significativa.

d) Productividad del gasto público.

Con objeto de medir la productividad del gasto público, se estimaron las siguientes funciones de producción: 


$$
Y_{t}^{F}=\alpha \mathrm{K}_{\mathrm{p}_{\mathrm{t}}}^{\beta} \mathrm{K}_{\mathrm{G}_{\mathrm{t}}}^{\gamma}, \mathrm{Y}_{\mathrm{t}}^{\mathrm{F}}=\beta \mathrm{K}_{\mathrm{p}_{\mathrm{t}}}+\not \mathrm{K}_{\mathrm{G}_{\mathrm{t}}}+\alpha
$$

El ingreso potencial $Y^{F}$ se estimó en base al mismo análisis de tendencia de la relación capital /producto usada anteriormente. Los acervos de capital público y privado se estimaron a partir de los datos proporcionados por el Banco de México; restando a la inversión total la inversión pública autorizada, se obtuvo la inversión privada. ${ }^{1}$. Los resultados de las regresiones son:

$$
\begin{array}{ccc}
\log \mathrm{Y}_{\mathrm{t}}^{\mathrm{F}}=1.38+.33 \operatorname{LogK}_{\mathrm{p}_{\mathrm{t}}}+.45 \operatorname{LogK}_{\mathrm{G}_{\mathrm{t}}} & R^{2}=.999 \\
(.029) & (.029) & \\
\mathrm{Y}_{\mathrm{t}}^{\mathrm{F}}=37363+1.08 \mathrm{~K}_{\mathrm{p}_{\mathrm{t}}}+.19 \mathrm{~K}_{\mathrm{G}_{\mathrm{t}}} & R^{2}=.995
\end{array}
$$

Como puede observarse, los dos tipos de ecuaciones dan resultados bastante diferentes. Sin embargo, la versión lo garítmica permite apreciar mejor el alto grado de complementalidad que probablemente existe entre la inversión pública y la privada. De cualquier modo, el altísimo grado de colinealidad entre estas variables plantea serias dudas sobre la validez de la estimación y, por consiguiente, sobre la magnitud real de los coeficientes.

IV.4 Análisis de sensibilidad.

a) Diseño del análisis.

Una conclusión general que se puede inferir de las estimaciones realizadas previamente, es que no es posible obtener valores muy seguros, ni de las elasticidades de las funciones, ni de la productividad del gasto público. Resulta por lo tanto indispensable realizar un análisis de sensibilidad. En esta sección consideraremos tres valores de los parámetros $c^{\prime} / i^{\prime}, \mathrm{d}^{\prime} / i^{\prime}$ y $\mu$ : un valor más "probable", un valor "alto" y un valor "bajo", estimados en base a las siguientes consideraciones:

1/ Este método está sujeto a críticas por varias razones. Primero porque se debería considerar la inversión realizada en vez de la autorizada. Segundo porque los datos de inversión pública incluyen la mayor parte del sector paraestatal. 
Se encontró que para $c^{\prime}$ el valor de .46 constituye seguramente un límite superior. Por otro parte, se observó que un valor de .20 para la elasticidad de la inversión con respecto al crédito sería probablemente un límite inferior. Esta elasticidad, dados los valore promedio de $I$ y de $\mathrm{L}$, nos da una valor para $i^{\prime}$ de aproximadamente .12. Ajustando $c^{\prime}$ par que corresponda al concepto de ingreso disponible en vez del ingreso total $\stackrel{1 /}{ }$, obtenemos un valor "alto" de $c^{\prime} / i^{\prime}$ de 4.3 . El valor "bajo" de $c^{\prime} / i^{\prime}$ podría ser cero, dado que el crédito no tenía ningún efecto sobre el consumo cuando se corrió la regresión en primeras diferencias. Un valor "intermedio" sería entonces de alrededor de $2.1^{21}$

Tomando como valor "intermedios", la relación de los valores medios de $d$ ' e i', se obtiene una cifra de alrededor de .084. Si por otra parte, se añade al coeficiente de $L_{t}$ la desviación estandar que resulta de la estimación de la ecuación en primeras diferencias, se obtiene una valor de .042 que puede considerarse un valor "alto" para $d$ '. Tomando .12 como un valor bajo para $i$ ', la estimación "alta" de $d^{\prime} / i$ ' sería de .35 ; la estimación "baja” podría ser cero.

Finalmente, para $\mu$, se calculó primero un porcentaje de inversión productiva ${ }^{3 /}$ dentro del gasto público federal total, obteniéndose una cifra de .32. Para las productividades comparadas de las inversiones pública y privada se tomaron como valores $1.50,1.30$ y .80 . El segundo valor es una relación de los coeficientes estandarizados de la ecuación logarítmica de ajuste de la función de producción. Considerando este valor como "intermedio", pero quizá un poco alto, se definieron límites máximos y mínimos de 1.5 y .8 respectivamente. Los valores correspondientes de $\mu$ son entonces de $.48, .42$ y .25 .

I/ $\quad C$ fue definido como $c Y d$ y I como iY por lotanto $c^{\prime} / i^{\prime}$ es $\left(\mathrm{C}^{\prime} / I^{\prime}\right) \times(\mathrm{Y} / \mathrm{Yd})$.

21 Este valor "probable" es más o menos consistente con las correlaciones que existen entre las desviaciones con respecto a las tendencias históricas de las variables $L$ y $\mathrm{C}, \mathrm{L}$ e I. Las primeras tienen una correlación de .29, mientras que $L$ e I tienen solamente una de .13 .

3/ Se tomaron en cuenta exclusivamente las inversiones del gobierno federa, descontando las inversiones en los sectores de petróleo, electricidad y otras industrias. Se hizo además un ajuste para tomar en cuenta la discrepancia promedio que existe entre la inversión autorizada y la realizada. 
b) Regiones y valores críticos.

Atendiendo a los diferentes valores de los parámetros $c^{\prime} / i^{\prime}, \mathrm{d}^{\prime} / i^{\prime}$ y $\mu$, se obtuvieron valores críticos que permitieron ubicar a la economía dentro de la región característica. Los resultados aparecen en el Cuadro 3. Los cambios en los valores de $\mathrm{d}^{\prime} / i^{\prime}$ no afectaron los resultados.

Cuadro 3

\begin{tabular}{rlll}
\hline & & 2.1 & 4.3 \\
.25 & II & II & III \\
.41 & II & III & III \\
.48 & II & III & III \\
\hline
\end{tabular}

Como se puede observar, la economía, dependiendo de los valores de $c^{\prime} / i^{\prime}$ y $\mu$ se encuentran en la región II o en la región III.

c) Cambios en la tasa de crecimiento.

Reduciendo a cero el flujo de encaje y suponiendo que fuera posible compensar esta disminución mediante un cambio en la recaudación fiscal, de tal manera que ni 1 ni $\lambda$ se vean afectados, se obtuvieron los cambios en la tasa de crecimiento indicados en el Cuadro 4.

Cuadro 4

\begin{tabular}{rrrr}
\hline$i$ \\
\hline 0
\end{tabular}

Los cambios en $d_{g}$ obtenidos son independientes de los valores de $\mu$. Los incrementos en $t$ necesarios para compensar la eliminación del encaje fueron del orden de un $12 \%$ en todos los 
casos. Como se puede observar, los incrementos porcentuales de la tasa de crecimiento real varían

entre 1 y 7\%. Cuando menores sean $c^{\prime} / i^{\prime}$ y $\mathrm{d}^{\prime} / i^{\prime}$, mayor será el crecimiento del ingreso. Este resultado es evidente: si la elasticidad de la inversión con respecto al crédito es muy alta en comparación con las elasticidades que tienen el consumo y el flujo internacional de capitales, con respecto al crédito al crédito, el encaje restringe en gran medida, principalmente a la inversión. $\mathrm{Su}$ supresión eleva notablemente la tasa de crecimiento.

Se puede notar que el posible efecto sobre la tasa de crecimiento en ningún caso rebasa el $.5 \%$ en cifras absolutas. La eliminación del endeudamiento interno tendría por lo tanto un impacto positivo, pero no espectacular sobre la tasa de crecimiento. Sin embargo, incluso un incremento inferior al $.5 \%$ puede traducirse en una notable diferencia de ingreso per capita después de algunos años.

d) Cambios en el endeudamiento externo.

Eliminando el encaje y manteniendo constantes tanto $g$ como $\lambda$ (lo que requiere un aumento del orden del $58 \%$ en la imposición fiscal) permitiría reducir en todos los casos la deuda externa en .76, o sea que permitiría, de hecho, eliminarla totalmente. Sin embargo, el grado de libertad que existe en el modelo no permite apreciar el realismo de este resultado: está, de hecho, sujeto a que sea posible eliminar totalmente el déficit en cuenta corriente y es probable que esto sea casi imposible a menos que se altere radicalmente la tasa de cambio.

e) Cambios en la participación del gobierno.

Los resultados sobre los movimientos en $\lambda$ como g y 1 constantes reflejan lo siguiente: cuando la economía está en la región II la eliminación del encaje debe ser acompañada de una substanciosa reducción en la imposición fiscal para que $\quad g$ y 1 puedan permanecer en su nivel original. $\lambda$ también sufre fuertes reducciones. Cuando la economía está en la región III la eliminación del encaje, acompañada de un muy pequeño aumento en la presión fiscal permite mantener $\lambda$ prácticamente constante, al igual que $g$ y 1 . 
IV.5 El caso en que los impuestos afectan la inversión.

En el caso extremo en que la inversión privada es proporcional al ingreso disponible en lugar de ser proporcional al ingreso total, se obtuvieron los resultados siguientes: la eliminación del encaje y un aumento de la presión fiscal del orden de un $11 \%$, dejando constantes $\lambda$ y 1 , reducen $g$ en un máximo de $.05 \%$, o sea que prácticamente no tiene impacto sobre la tasa de crecimiento. Manteniendo $g$ y $\lambda$ constantes, la eliminación del encaje junto con un aumento del orden de $10 \%$ de los impuestos incrementa la deuda externa en un máximo de $9 \%$. Finalmente, dejando $g$ y 1 constantes, la eliminación del encaje, junto con pequeñas variaciones en la tasa de imposición fiscal (positivas o negativas según los casos), permite hacer aumentar o reducir ligeramente la participación del estado. La conclusión evidente en este caso extremo es entonces que la eliminación del encaje y su substitución por impuestos no tendría prácticamente efecto alguno sobre la economía.

\section{Conclusiones.}

En este trabajo se ha intentado evaluar las implicaciones que tendría un cambio importante en los canales de financiamiento del gasto público en México. La principal conclusión que se obtiene a partir de estas estimaciones, es que prácticamente en todos los casos el reemplazo total del endeudamiento interno por una mayor recaudación fiscal, tendría efectos benéficos sobre la tasa de crecimiento, conservando constantes la deuda externa y la participación del estado en la economía. El impacto sobre ésta no sería espectacular; el crecimiento no se incrementaría en más de medio punto de porcentaje, sin embargo, sí sería significativo en un contexto de largo plazo. Es probable que, por otra parte, una alta tasa de encaje conduzca a una mayor concentración industrial y financiera y, por ende, a mayores desequilibrios estructurales en la economía así como a un empeoramiento de la distribución del ingreso. La gradual substitución del encaje por impuestos parece entonces ser deseable, tanto por que aceleraría el proceso de crecimiento como porque tendría un impacto estructural probablemente favorable. 


\section{BIBLIOGRAFÍA}

Brothers D. Y L. Solís (1968)

Hicks, J. (1967)

Ize, A. (1878)

Montemayor, R. (1976)

Ortiz, G. (1977)
Mexican Financial Development.

University of Texas Press

Capital and Growth.

Oxford University Press.

"Un Análisis de la Inflación en México".

Documento de Trabajo. El Colegio de México.

An Econometric Model of the Financial Sector in Mexico.

Tesis Doctoral, University of Pennsylvania.

Capital Accumulation and Economic Growth:

A Financial Perspective on Mexico.

Tesis Doctoral, Stanford University.

Versión en Español a publicarse próximamente por el CEMLA. 